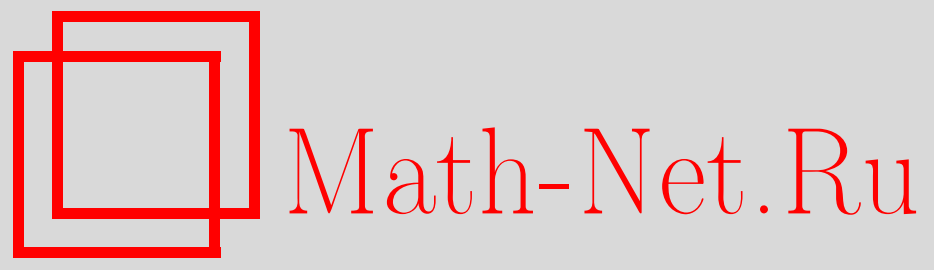

Н. П. Салихов, Оптимальные последовательности критериев для различения нескольких полиномиальных схем испытаний, Теория вероятн. и ее примен., 2002, том 47, выпуск 2, 270-285

DOI: https://doi.org/10.4213/tvp3647

Использование Общероссийского математического портала MathNet.Ru подразумевает, что вы прочитали и согласны с пользовательским соглашением

http: //www . mathnet.ru/rus/agreement

Параметры загрузки:

IP : 3.85 .183 .62

26 апреля 2023 г., $16: 31: 18$

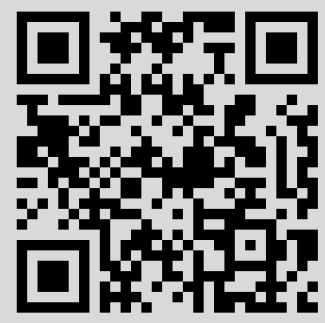




\title{
ОПТИМАЛЬНЫЕ ПОСЛЕДОВАТЕЛЬНОСТИ КРИТЕРИЕВ ДЛЯ РАЗЛИЧЕНИЯ НЕСКОЛЬКИХ ПОЛИНОМИАЛЬНЫХ СХЕМ ИСПЫТАНИЙ
}

\begin{abstract}
Рассматриваются последовательности $\left\{\omega^{n}\right\}$ критериев $\omega^{n}$ для решения задачи выбора истинной полиномиальной схемы испытаний по частотам исходов при $n$ независимых испытаниях, произведенных по одной из $m$ возможных схем с одним и тем же множеством исходов. Пусть $\alpha_{s}\left(\omega^{n}\right)$ - вероятность не принять истинную $s$-ю схему, $s=1, \ldots, m$. Изучается поведение величины $\max _{s \in J} \alpha_{s}\left(\omega^{n}\right)$ при заданном $J \subseteq\{1, \ldots, m\}$ и $n \rightarrow \infty$ для последовательностей $\left\{\omega^{n}\right\}$ из множества $N$, характеризующегося тем, что вероятности $\alpha_{t}\left(\omega^{n}\right)$ при $t \in I, I \subseteq\{1, \ldots, m\}$, удовлетворяют некоторым условиям, например, $\alpha_{t}\left(\omega^{n}\right) \leqslant \alpha_{t}<1$ или $\alpha_{t}\left(\omega^{n}\right) \leqslant a_{t} \exp \left(-n v_{t}\right)$ при всех $n \geqslant n_{0}$. Указаны последовательности $\left\{g^{n}\right\} \in N$ и явно вычислена величина $M(N, J) \geqslant 0$ такая, что $\max _{s \in J} \alpha_{s}\left(g^{n}\right)=\exp (-n M(N, J)+o(n))$ и не существует последовательности $\left\{\omega^{n}\right\} \in N$, для которой $\max _{s \in J} \alpha_{s}\left(\omega^{n}\right)=$ $\exp (-n M+o(n)), M>M(N, J)$. Явно вычислены стремящиеся к 0 при $n \rightarrow \infty$ верхние границы для $\alpha_{t}\left(g^{n}\right), t=1, \ldots, m$.
\end{abstract}

Ключевые слова и фразы: полиномиальная схема испытаний, различение нескольких простых гипотез, оптимальные последовательности критериев, расстояние Кульбака-Лейблера, расстояние Чернова.

1. Введение. Задача о различении нескольких простых статистических гипотез является естественным обобщением классической задачи о различении двух простых гипотез. При различении нескольких альтернативных гипотез возникает задача идентификации (см. $[14, \S 7 \mathrm{~d}])$. Важность многоальтернативной задачи отмечалась, например, в монографиях [2, с. 167-168], [12, предисловие]. Одной из первых работ по многоальтернативной задаче является [13]. Задача о различении конечного числа законов распределения была поставлена А. Н. Колмогоровым в 1940 году, а изложенные в [13] результаты получены в 1941-1942 годах. Из ранних работ обзорного характера, в которых имеются постановки основных проблем в многоальтернативной задаче, можно отметить [26], $[31$, гл. 6]. В [7], [10] (выбор из многомерных нормальных законов), [13],

\footnotetext{
*ФАПСИ, Б. Кисельный пер., 4, 103031 Москва, Россия.
} 
[23], [25], [40] вводятся различные определения оптимального решающего правила, изучаются свойства и способы его построения.

Важные результаты получены по следующим задачам.

А. Найти оценки для компонент вектора потерь или для некоторых функций от его компонент (см. [1], [5], [9], [21, гл. 4], [22], [41], [42], [46]).

Б. Исследовать поведение компонент вектора потерь или некоторых функций от его компонент с ростом числа испытаний (см. [6], [38], $[44],[47])$.

Характерной чертой многих ранних работ по этим задачам в многоальтернативном случае является поиск оценок сверху для среднего байесовского риска с помощью некоторых выражений типа энтропии Шеннона, а затем исследование поведения этих оценок. В [16] для задачи Б исследовано поведение компонент вектора потерь в простейшем случае, когда вектор потерь совпадает с вектором вероятностей ошибок. В случае двух альтернатив такой подход применялся в $[11$, гл. $4, \S 3],[14$, $\S 7$ а.3], [19], [21, гл. 4, § 4.4], [24], [27]-[30], [35], [37], [45].

В настоящей статье изучается поведение вероятностей ошибок критериев, применяемых для решения следуюшей задачи идентификации: по реализации конечной последовательности независимых полиномиальных испытаний определить, какому из заданных полиномиальных распределений она соответствует.

Пусть гипотеза $H_{t}, t \in\{1, \ldots, m\}, m \geqslant 2$, состоит в том, что последовательность $b_{1}, b_{2}, \ldots$ является последовательностью независимых испытаний по полиномиальной схеме с исходами $1, \ldots, r(r \geqslant 2)$, вероятности появления которых при каждом испытании равны соответственно $p_{t, 1}, \ldots, p_{t, r}$. Предполагается, что $\mathbf{p}_{t}=\left(p_{t, 1}, \ldots, p_{t, r}\right) \in \Omega_{+}$для $t=1, \ldots, m$, где $\Omega_{+}=\Omega_{+}(r)=\left\{\mathbf{u}=\left(u_{1}, \ldots, u_{r}\right): u_{1}>0, \ldots, u_{r}>0\right.$, $\left.u_{1}+\cdots+u_{r}=1\right\}$, и что все векторы $\mathbf{p}_{1}, \ldots, \mathbf{p}_{m}$ различны. Наблюдения $b_{1}, \ldots, b_{n}, n \geqslant 1$, будем характеризовать точкой $\underline{\mathbf{x}}=\left(\underline{x}_{1}, \ldots, \underline{x}_{r}\right)=$ $\left(x_{1} / n, \ldots, x_{r} / n\right)$, где $x_{k}$ - число появлений исхода $k \in\{1, \ldots, r\}$ среди $b_{1}, \ldots, b_{n}$. Пусть $\Omega_{n}$ - пространство значений вектора $\underline{\mathbf{x}},\left|\Omega_{n}\right|=C_{n+r-1}^{r-1}$, соответствующих $n$ наблюдениям. Здесь и далее $|A|$ - мощность множества $A, \operatorname{Ind}(A)$ - индикатор события $A$. Гипотеза $H_{t}$ определяет на $\Omega_{n}$ вероятностную меру

$$
\mathbf{P}_{n}\left(\underline{\mathbf{x}}, \mathbf{p}_{t}\right)=\frac{n !}{x_{1} ! \cdots x_{r} !} p_{t, 1}^{x_{1}} \cdots p_{t, r}^{x_{r}} .
$$

Для любых $\mathbf{p}=\left(p_{1}, \ldots, p_{r}\right) \in \Omega_{+}$и множества $\omega \subseteq \Omega$, где

$$
\Omega=\Omega(r)=\left\{\mathbf{u}=\left(u_{1}, \ldots, u_{r}\right): u_{1} \geqslant 0, \ldots, u_{r} \geqslant 0, u_{1}+\cdots+u_{r}=1\right\},
$$

положим $\mathbf{P}_{n}(\omega, \mathbf{p})=\sum_{\underline{\mathbf{x}} \in \omega \cap \Omega_{n}} \mathbf{P}_{n}(\underline{\mathbf{x}}, \mathbf{p})$, если $\omega \cap \Omega_{n} \neq \varnothing$, и $\mathbf{P}_{n}(\omega, \mathbf{p})=0$, если $\omega \cap \Omega_{n}=\varnothing$. В дальнейшем для оценок вероятностей ошибок критериев важную роль будут играть оценки вероятностей $\mathbf{P}(\omega(\mathbf{a}, \mathbf{b}, c), \mathbf{p})$, 
где

$$
\begin{gathered}
\omega(\mathbf{a}, \mathbf{b}, c)=\left\{\mathbf{u}=\left(u_{1}, \ldots, u_{r}\right): \mathbf{u} \in \Omega, \sum_{k=1}^{r} u_{k} \ln \frac{a_{k}}{b_{k}} \geqslant c\right\}, \\
\mathbf{a}=\left(a_{1}, \ldots, a_{r}\right) \in \Omega_{+}, \quad \mathbf{b}=\left(b_{1}, \ldots, b_{r}\right) \in \Omega_{+}, \quad \mathbf{a} \neq \mathbf{b}, \quad c \in \mathbf{R} .
\end{gathered}
$$

Для любых $\mathbf{p}=\left(p_{1}, \ldots, p_{r}\right) \in \Omega_{+}$и $\mathbf{u}=\left(u_{1}, \ldots, u_{r}\right) \in \Omega$ пусть $I(\mathbf{u}, \mathbf{p})=$ $\sum_{k=1}^{r} u_{k} \ln \left(u_{k} / p_{k}\right)$ - расстояние Кульбака-Лейблера от $\mathbf{u}$ до $\mathbf{p}$. Известно, что $I(\mathbf{u}, \mathbf{p}) \geqslant 0$ и что равенство имеет место только при $\mathbf{u}=\mathbf{p}$ (см., например, [11, гл. 2, §3]).

Пусть $A_{n}^{0}$ - множество всех разбиений $\omega^{n}=\left(\omega_{0}^{n}, \omega_{1}^{n}, \ldots, \omega_{m}^{n}\right)$ множества $\Omega_{n}$ на $m+1$ попарно не пересекающихся множеств $\omega_{t}^{n}$ (некоторые $\omega_{t}^{n}$ могут быть пустыми) и

$$
A^{0}=\left\{\left(\omega^{1}, \omega^{2}, \ldots\right): \omega^{n} \in A_{n}^{0} \text { для } n \geqslant 1\right\}
$$

Каждое разбиение $\omega^{n}$ определяет критерий различения альтернативных гипотез $H_{1}, \ldots, H_{m}$, основанный на испытаниях $b_{1}, \ldots, b_{n}$ : если точка $\underline{\mathbf{x}}$, характеризующая испытания $b_{1}, \ldots, b_{n}$, принадлежит $\omega_{t}^{n}$, то при $t \in\{1, \ldots, m\}$ принимается гипотеза $H_{t}$, а при $t=0$ не принимается ни одна из гипотез. При $s \in\{1, \ldots, m\}$ величина

$$
\alpha_{s}\left(\omega^{n}\right)=1-\mathbf{P}_{n}\left(\omega_{s}^{n}, \mathbf{p}_{s}\right)=\sum_{t \in\{0,1, \ldots, m\} \backslash\{s\}} \mathbf{P}_{n}\left(\omega_{t}^{n}, \mathbf{p}_{s}\right)
$$

является вероятностью ошибки $s$-го рода (т.е. вероятностью не принять истинную гипотезу $H_{s}$ ) при применении разбиения (критерия) $\omega^{n}$.

Для любой последовательности критериев $\left\{\omega^{n}\right\}=\left(\omega^{1}, \omega^{2}, \ldots\right) \in A^{0}$ рассмотрим поведение при $n \rightarrow \infty$ величины $\max _{s \in J} \alpha_{s}\left(\omega^{n}\right)$, где $J \subseteq$ $\{1, \ldots, m\}, J \neq \varnothing$.

О п р е д е л е н и е $1 . \quad J$-мерой асимптотической эффективности для последовательности $\left\{\omega^{n}\right\} \in A^{0}$ назовем величину $M\left(\left\{\omega^{n}\right\}, J\right)=$ $-\liminf _{n \rightarrow \infty} T\left(\omega^{n}, J\right)$, где $T\left(\omega^{n}, J\right)=-\infty$, если $\max _{s \in J} \alpha_{s}\left(\omega^{n}\right)=0$, и $T\left(\omega^{n}, J\right)=n^{-1} \ln \max _{s \in J} \alpha_{s}\left(\omega^{n}\right)$, если $\max _{s \in J} \alpha_{s}\left(\omega^{n}\right)>0$.

Величина $T\left(\omega^{n}, J\right)=-\infty$ только в тех случаях, когда $J=\{t\}$ при некотором $t \in\{1, \ldots, m\}, \omega_{t}^{n}=\Omega_{n}$. Для случая $m=2,|J|=1$ величина $M\left(\left\{\omega^{n}\right\}, J\right)$ рассматривалась в [37], [45].

О п р е д е л е н и е 2 . Пусть $N \subseteq A^{0}$. Назовем последовательность $\left\{g^{n}\right\} \in N J$-оптимальной последовательностью (для) множества $N$, если

$$
M\left(\left\{g^{n}\right\}, J\right)=-\lim _{n \rightarrow \infty} T\left(g^{n}, J\right)=M(N, J) \stackrel{\text { def }}{=} \sup _{\left\{\omega^{n}\right\} \in N} M\left(\left\{\omega^{n}\right\}, J\right) .
$$

Укажем простые свойства $J$-оптимальных последовательностей. 
С в о й с т в о 1. Если последовательность $\left\{g^{n}\right\}$ является $J$-оптимальной для $N,\left\{\omega^{n}\right\} \in N, M\left(\left\{\omega^{n}\right\}, J\right)<M(N, J)$, то справедливо соотношение $\lim _{n \rightarrow \infty} \max _{s \in J} . \alpha_{s}\left(g^{n}\right) / \max _{s \in J} \alpha_{s}\left(\omega^{n}\right)=0$.

С в о й с т в о 2. Если $N \subseteq N^{0} \subseteq A^{0}$, последовательность $\left\{g^{n}\right\} \in N$ и $J$-оптимальна для $N^{0}$, то она $J$-оптимальна и для $N$.

С в о й с т в о 3 . Если последовательность $\left\{g^{n}\right\} \in N$ и число $b$ таковы, что

$$
M(N, J) \leqslant b \leqslant-\limsup _{n \rightarrow \infty} T\left(g^{n}, J\right),
$$

то $\left\{g^{n}\right\}$ является $J$-оптимальной для $N$ и $M(N, J)=b$.

Нашей целью будет вычисление величин $M(N, J)$, построение $J$-оптимальных последовательностей $\left\{g^{n}\right\}$ для $N=A^{0}$ и для

$$
\begin{aligned}
& N=P=\left\{\left\{\omega^{n}\right\} \in A^{0}: \lim _{n \rightarrow \infty} n^{-1} \ln \left(1-\alpha_{t}\left(\omega_{t}^{n}\right)\right)=0 \text { для } t \in I\right\}, \\
& N=P_{*}=\left\{\left\{\omega^{n}\right\} \in A^{0}: \alpha_{t}\left(\omega_{t}^{n}\right) \leqslant \alpha_{t}, \alpha_{t} \in(0,1) \text { для } t \in I, n \geqslant n_{0}\right\}, \\
& N=P_{1}=\left\{\left\{\omega^{n}\right\} \in A^{0}: \lim _{n \rightarrow \infty} \alpha_{t}\left(\omega_{t}^{n}\right)=0 \text { для } t \in I\right\}, \\
& N=P_{0}=\left\{\left\{\omega^{n}\right\} \in A^{0}: \alpha_{t}\left(\omega^{n}\right) \leqslant a_{t} \exp \left(-n v_{t}\right), a_{t}>0, v_{t}>0,\right. \\
& \text { для } t \in I, n \geqslant 1\},
\end{aligned}
$$

где $I \subseteq\{1, \ldots, m\}, I \neq \varnothing$ (зависимость от параметров $I, \alpha_{t}, n_{0}, a_{t}, v_{t}$ в явном виде в обозначениях $P, P_{*}, P_{1}, P_{0}$ не указываем).

Последовательности из $P_{*}$ естественно рассматривать, когда гипотезы $H_{t}$ при $t \in I=\{1, \ldots, m\} \backslash J$ появляются редко (пусть сумма априорных вероятностей этих гипотез не более малого числа $\varepsilon$ ).

Действительно, в этом случае средний байесовский риск (при простейшем виде убытка от ложной идентификации, см. $[14, \S 7 \mathrm{~d}]$ ) оценивается сверху величиной $(1-\varepsilon) \max _{s \in J} \alpha_{s}\left(\omega^{n}\right)+\varepsilon \max _{t \in I} \alpha_{t}$, и естественно использовать такую последовательность $\left\{\omega^{n}\right\} \in P_{*}$, для которой величина $\max _{s \in J} \alpha_{s}\left(\omega^{n}\right)$ убывает при $n \rightarrow \infty$ с максимально большой скоростью $M\left(P_{*}, J\right)$.

В [18], [33], [39] величина $M(N, J)$ была вычислена лишь для случая $J=\{1, \ldots, m\}, N=A^{0}$. Основные результаты о множествах $P, P_{1}$ были опубликованы без доказательств в [16]. В теоремах 3,4 (п. 3) найдены новые по сравнению с [16] $J$-оптимальные последовательности для $P, P_{1}$. В теоремах 3,5 содержатся новые результаты о множестве $P_{0}$, обобщающие на случай $m>2$ результаты из [24], [29]. Кроме того, здесь получены явные и стремящиеся к 0 при $n \rightarrow \infty$ верхние оценки вероятностей ошибок $s$-го рода, возникающих при применении критериев из $J$-оптимальных последовательностей. Все эти критерии являются байесовскими. Они строятся на основе доказанного в 1939 году в [48] 
обобщения на случай многих альтернатив известной (1933 года) леммы Неймана-Пирсона (см. также [14, §7a], [31, гл. 6], [36]).

При любом $\lambda=\left(\lambda_{1}, \ldots, \lambda_{m}\right) \in \Omega(m)$ определим (см. [14, §7d.3]) $\lambda$-байесовский (байесовский) критерий $\omega^{n}(\lambda)=\left(\omega_{0}^{n}(\lambda), \omega_{1}^{n}(\lambda), \ldots, \omega_{m}^{n}(\lambda)\right)$, в котором каждая точка $\underline{\mathbf{x}} \in \Omega_{n}$ включается в одно из множеств $\omega_{t}^{n}(\lambda)$, $t \in\{1, \ldots, m\}, \lambda_{t}>0$, если

$$
\lambda_{t} \mathbf{P}_{n}\left(\underline{\mathbf{x}}, \mathbf{p}_{t}\right)=\max _{1 \leqslant i \leqslant m} \lambda_{i} \mathbf{P}_{n}\left(\underline{\mathbf{x}}, \mathbf{p}_{i}\right)
$$

(при этом $\omega_{0}^{n}(\lambda)=\varnothing, \omega_{t}^{n}(\lambda)=\varnothing$, если $\left.\lambda_{t}=0\right)$.

Из (3) следует, что при $t, s \in\{1, \ldots, m\}, t \neq s, \lambda_{t}>0, \lambda_{s}>0$

$$
\omega_{t}^{n}(\lambda) \subseteq \omega\left(\mathbf{p}_{t}, \mathbf{p}_{s}, n^{-1} \ln \frac{\lambda_{s}}{\lambda_{t}}\right)
$$

2. Оценки вероятностей $\mathbf{P}_{n}(\omega(\mathbf{a}, \mathbf{b}, c), \mathbf{p})$. Приведем необходимые для оценивания сверху вероятностей ошибок $s$-го рода (и в основном известные) верхние оценки определенных в п. 1 вероятностей $\mathbf{P}_{n}(\omega(\mathbf{a}, \mathbf{b}, c), \mathbf{p})$ при $\mathbf{a} \neq \mathbf{b}$. Для этого введем величины

$$
\begin{gathered}
E_{t}=E_{t}(\mathbf{p}, \mathbf{a}, \mathbf{b})=\sum_{k=1}^{r} p_{k}\left(\ln \frac{a_{k}}{b_{k}}\right)^{t}, \quad t=1,2, \\
\theta_{1}=\theta_{1}(\mathbf{a}, \mathbf{b})=\min _{1 \leqslant k \leqslant r} \ln \frac{a_{k}}{b_{k}}, \quad \theta_{2}=\theta_{2}(\mathbf{a}, \mathbf{b})=\max _{1 \leqslant k \leqslant r} \ln \frac{a_{k}}{b_{k}}
\end{gathered}
$$

и функции

$$
\begin{aligned}
\gamma(c, \mathbf{a}, \mathbf{b}, \mathbf{p}) & =\min _{\mathbf{u} \in \omega(\mathbf{a}, \mathbf{b}, c)} I(\mathbf{u}, \mathbf{p}), \quad c \in\left[E_{1}, \theta_{2}\right] \\
\gamma(c) & =\gamma(c, \mathbf{a}, \mathbf{p})=\gamma(c, \mathbf{a}, \mathbf{p}, \mathbf{p}), \quad c \in\left[-I(\mathbf{p}, \mathbf{a}), \theta_{2}(\mathbf{a}, \mathbf{p})\right], \\
V(d) & =V(d, \mathbf{a}, \mathbf{p})=\min _{\mathbf{u} \in \Omega: I(\mathbf{u}, a) \leqslant d} I(\mathbf{u}, \mathbf{p}), \quad d \geqslant 0
\end{aligned}
$$

$(V(d)=0$ при $d \geqslant I(\mathbf{p}, \mathbf{a}))$.

Лемма 1. Если $Y_{1}, \ldots, Y_{n}$ - независимые случайные величинь, $\mathbf{E} Y_{k}=0, Y_{k} \leqslant b \partial_{\Omega s} k=1, \ldots, n, d=n^{-1} \sum_{k=1}^{n} \mathbf{D} Y_{k}, 0<\varepsilon \leqslant b$, mo

$$
\mathbf{P}\left\{\sum_{k=1}^{n} Y_{k} \geqslant n \varepsilon\right\}<\exp \left(-\frac{n \varepsilon^{2}}{2(d+b \varepsilon)}\right) \leqslant \exp \left(-\frac{n \varepsilon^{2}}{2\left(d+b^{2}\right)}\right) .
$$

Д о к а за т е л ь с т в о. При любом $\varepsilon>0$ верно неравенство Беннета [34]

$$
\mathbf{P}\left\{\sum_{k=1}^{n} Y_{k} \geqslant n \varepsilon\right\} \leqslant \exp \left(-\frac{n d}{b^{2}} f(y)\right)
$$


где $y=b \varepsilon / d, f(y)=(1+y) \ln (1+y)-y$. По формуле Тейлора для любого $y>0$ при некотором $\delta \in(0,1)$

$$
f(y)=\frac{f^{\prime \prime}(\delta y)}{2}=\frac{y^{2}}{2(1+\delta y)}>\frac{y^{2}}{2(1+y)} .
$$

Отсюда и из неравенства Беннета получим утверждение леммы.

Лемма 2. Справедливь следующие утверждения.

A. Ecли $c \in\left[E_{1}, \theta_{2}\right]$, mo

$$
\begin{gathered}
\mathbf{P}_{n}(\omega(\mathbf{a}, \mathbf{b}, c), \mathbf{p}) \leqslant \exp (-n \gamma(c, \mathbf{a}, \mathbf{b}, \mathbf{p})), \\
\lim _{n \rightarrow \infty} n^{-1} \ln \mathbf{P}_{n}(\omega(\mathbf{a}, \mathbf{b}, c), \mathbf{p})=-\gamma(c, \mathbf{a}, \mathbf{b}, \mathbf{p}),
\end{gathered}
$$

$2 \partial e$

$$
\gamma(c, \mathbf{a}, \mathbf{b}, \mathbf{p})>0 \quad n p u c \in\left(E_{1}, \theta_{2}\right]
$$

$\gamma\left(E_{1}, \mathbf{a}, \mathbf{b}, \mathbf{p}\right)=0, \quad \gamma\left(\theta_{2}, \mathbf{a}, \mathbf{b}, \mathbf{p}\right)=-\ln \left(\sum_{k=1}^{r} p_{k} \operatorname{Ind}\left(\ln \frac{a_{k}}{b_{k}}=\theta_{2}\right)\right)$.

B.

$$
\mathbf{P}_{n}\left(\omega\left(\mathbf{a}, \mathbf{b}, E_{1}+\varepsilon\right), \mathbf{p}\right) \leqslant \exp \left(-n \varepsilon^{2} h(\mathbf{a}, \mathbf{b}, \mathbf{p})\right)
$$

где либо $\varepsilon>0$,

$$
h(\mathbf{a}, \mathbf{b}, \mathbf{p})=h_{0}(\mathbf{a}, \mathbf{b}, \mathbf{p})=\max \left(\frac{1}{2\left(E_{2}-2 \theta_{2} E_{1}+\theta_{2}^{2}\right)}, \frac{2}{\left(\theta_{2}-\theta_{1}\right)^{2}}\right),
$$

либо

$$
\begin{gathered}
\varepsilon \in\left(0, \frac{e^{2 \tau}-1-2 \tau}{\tau} \cdot \frac{E_{2}-E_{1}^{2}}{\max \left(\theta_{2}-E_{1}, E_{1}-\theta_{1}\right)}\right), \quad \tau>0, \\
h(\mathbf{a}, \mathbf{b}, \mathbf{p})=h_{1}(\mathbf{a}, \mathbf{b}, \mathbf{p})=\max \left(h_{0}(\mathbf{a}, \mathbf{b}, \mathbf{p}), \frac{\tau^{2}}{e^{2 \tau}-1-2 \tau} \cdot \frac{1}{E_{2}-E_{1}^{2}}\right) . \\
\text { C. Ecли } c \in[-I(\mathbf{p}, \mathbf{a}), I(\mathbf{a}, \mathbf{p})], \text { то верньи равенство } \\
\gamma(c, \mathbf{a}, \mathbf{p})=\gamma(-c, \mathbf{p}, \mathbf{a})+c
\end{gathered}
$$

и неравенства

$$
\begin{aligned}
\gamma(c, \mathbf{a}, \mathbf{p}) & \leqslant I(\mathbf{a}, \mathbf{p}), \\
\gamma(-c, \mathbf{p}, \mathbf{a}) & \leqslant I(\mathbf{p}, \mathbf{a})
\end{aligned}
$$

равенство в (9) и (10) достигается только при $c=I(\mathbf{a}, \mathbf{p}) u$ $c=-I(\mathbf{p}, \mathbf{a})$ соответственно. 
Ecлu $c \in\left[-I(\mathbf{p}, \mathbf{a}), \theta_{2}(\mathbf{a}, \mathbf{p})\right] \backslash\{I(\mathbf{a}, \mathbf{p})\}$, mo

$$
\gamma(c, \mathbf{a}, \mathbf{p})>c \text {. }
$$

D. Ecлu $c \in[-I(\mathbf{p}, \mathbf{a}), I(\mathbf{a}, \mathbf{p})], m o$

$$
\gamma(c)=V(\gamma(c)-c, \mathbf{a}, \mathbf{p})
$$

Д о к а з а т е л ь с в о. Справедливы следующие равенства:

$$
\mathbf{P}_{n}(\omega(\mathbf{a}, \mathbf{b}, c), \mathbf{p})=\mathbf{P}\left\{X_{1}+\cdots+X_{n} \geqslant n c\right\},
$$

где случайные величины $X_{1}, X_{2}, \ldots$ независимы и распределены так же, как случайная величина $X$, которая принимает значение $\ln \left(a_{k} / b_{k}\right)$ с вероятностью $p_{k}, k=1, \ldots, r$ (см. [20, § 8.3]). Применяя критерий оптимальности в задаче выпуклого программирования (см., например, [8, теорема 3.5.4]), можно при любом $c \in\left[E_{1}, \theta_{2}\right)$ вывести формулы

$$
\gamma(c, \mathbf{a}, \mathbf{b}, \mathbf{p})=\sigma \mu^{\prime}(\sigma)-\mu(\sigma)=\max _{x \in \mathbf{R}}(c x-\mu(x))=\max _{x \geqslant 0}(c x-\mu(x)),
$$

где $\mu(x)=\mu(x, \mathbf{a}, \mathbf{b}, \mathbf{p})=\ln \sum_{k=1}^{r} p_{k}\left(a_{k} / b_{k}\right)^{x}$,

$$
\mu^{\prime}(x)=\sum_{k=1}^{r} z_{k}(x) \ln \frac{a_{k}}{b_{k}}, \quad \mu^{\prime \prime}(x)=\sum_{k=1}^{r} z_{k}(x)\left(\mu^{\prime}(x)-\ln \frac{a_{k}}{b_{k}}\right)^{2},
$$

$z_{k}(x)=p_{k}\left(a_{k} / b_{k}\right)^{x} \exp (-\mu(x))$ для $k=1, \ldots, r, x \in \mathbf{R}, \sigma=\sigma(c)=$ $\sigma(c, \mathbf{a}, \mathbf{b}, \mathbf{p})$ - единственный неотрицательный корень уравнения $\mu^{\prime}(x)=$ $c ; \mu^{\prime}(0)=E_{1}, \sigma\left(E_{1}\right)=0$. При этом точка $\left(z_{1}(\sigma), \ldots, z_{r}(\sigma)\right)$ является граничной точкой множества $\omega(\mathbf{a}, \mathbf{b}, c)$ и единственной точкой минимума функции $I(\mathbf{u}, \mathbf{p})$ на нем.

А. Неравенство (5) доказано (для величин $X_{i}$ общего вида) в $[27$, теорема 1], равенство (5) следует из [35, теорема 6.1, лемма А.2] (см. также $[20, \S 8.3,8.5]$ или - для величин $X_{i}$ общего вида - $[3$, гл. $8, \S 8]$, $[4$, гл. $1, \S 1.7])$. У тверждения (5) верны также при замене в них $\omega(\mathbf{a}, \mathbf{b}, c)$ любым замкнутым выпуклым множеством $\omega \in \Omega$ (см. [18, леммы 1-3, теорема 2]). Утверждение (6) следует из свойства расстояния КульбакаЛейблера.

В. Оценка (7) с $h(\mathbf{a}, \mathbf{b}, \mathbf{p})=h_{0}(\mathbf{a}, \mathbf{b}, \mathbf{p})$ следует из (13), леммы 1 и неравенства Хефдинга [34]. Для доказательства оценки $(7)$ с $h(\mathbf{a}, \mathbf{b}, \mathbf{p})=$ $h_{1}(\mathbf{a}, \mathbf{b}, \mathbf{p})$ достаточно применить лемму 1 из [15].

С. Равенство (8) можно доказать с помощью (14) и тождества $\mu(x, \mathbf{p}, \mathbf{a}, \mathbf{a}) \equiv \mu(1-x, \mathbf{a}, \mathbf{p}, \mathbf{p})$.

Положим $\mu(x)=\mu(x, \mathbf{a}, \mathbf{p}, \mathbf{p}), \sigma=\sigma(c, \mathbf{a}, \mathbf{p}, \mathbf{p})$. Записав (14) в виде $\gamma\left(\mu^{\prime}(\sigma)\right)=\sigma \mu^{\prime}(\sigma)-\mu(\sigma)$ и дифференцируя это равенство, найдем: $\gamma^{\prime}\left(\mu^{\prime}(\sigma)\right)=\sigma, \gamma^{\prime \prime}\left(\mu^{\prime}(\sigma)\right) \mu^{\prime \prime}(\sigma)=1, \gamma^{\prime \prime}(c)=1 / \mu^{\prime \prime}(\sigma)>0$. Отсюда и из 
равенств $\gamma^{\prime}(-I(\mathbf{p}, \mathbf{a}))=0, \gamma(I(\mathbf{a}, \mathbf{p}))=I(\mathbf{a}, \mathbf{p})$ (которые следуют из (14) и равенств $\left.\mu(0)=0, \mu^{\prime}(0)=-I(\mathbf{p}, \mathbf{a}), \mu(1)=0, \mu^{\prime}(1)=I(\mathbf{a}, \mathbf{p})\right)$ получим (9). Неравенство (10) - простое следствие (9).

Докажем неравенство (11). Пусть $\omega=\omega(\mathbf{a}, \mathbf{p}, c)$. Тогда

$$
\gamma(c)=\min _{\mathbf{u} \in \omega} I(\mathbf{u}, \mathbf{p})=\min _{\mathbf{u} \in \omega}\left(I(\mathbf{u}, \mathbf{a})+\sum_{k=1}^{r} u_{k} \ln \frac{a_{k}}{p_{k}}\right) \geqslant \min _{\mathbf{u} \in \omega} \sum_{k=1}^{r} u_{k} \ln \frac{a_{k}}{b_{k}} \geqslant c,
$$

где оба неравенства превращаются в равенства только при $c=I(\mathbf{a}, \mathbf{p})$ (по свойству расстояния Кульбака-Лейблера).

D. Из свойства расстояния Кульбака-Лейблера следуют равенства: $V(0)=I(\mathbf{a}, \mathbf{p}), V(I(\mathbf{p}, \mathbf{a}))=0$. Отсюда и из следующих из (8), (9) paвенств $\gamma(-I(\mathbf{p}, \mathbf{a}))=0, \gamma(I(\mathbf{a}, \mathbf{p}))=I(\mathbf{a}, \mathbf{p})$ делаем вывод: D верно для крайних значений $c$. Пусть $c \in(-I(\mathbf{p}, \mathbf{a}), I(\mathbf{a}, \mathbf{p})), x=\gamma(c)-c$. Из (11), (8), (10) следует, что $x \in(0, I(\mathbf{p}, \mathbf{a}))$. Известно (см. [24, теоремы 4, 7], $\left[35\right.$, теорема 5.1]), что $V(d)=d+\mu^{\prime}(1-s)$ при $d \in(0, I(\mathbf{p}, \mathbf{a}))$, где $s-$ единственный на $(0,1)$ корень уравнения $-s \mu^{\prime}(1-s)-\mu(1-s)=d$. (Это можно доказать, применяя критерий оптимальности в задаче выпуклого программирования.) Из (14) следует, что при $d=x$ величина $s=1-\sigma$, где $\sigma=\sigma(c, \mathbf{a}, \mathbf{p}, \mathbf{p}), \mu^{\prime}(\sigma)=c$. Следовательно, $V(x)=x+\mu^{\prime}(\sigma)=\gamma(c)$.

3 а м е ч а н и е 1 . При $n \geqslant n^{*}, c \in\left[E_{1}, \theta_{2}\right)$ оценку в (5) можно уточнить: $\mathbf{P}_{n}(\omega(\mathbf{a}, \mathbf{b}, c), \mathbf{p}) \leqslant G n^{-1 / 2} \exp (-n \gamma(c, \mathbf{a}, \mathbf{b}, \mathbf{p}))$ (доказательство с указанием явного вида констант $n^{*}, G$ имеется в [17]).

При $\mathbf{b}=\mathbf{p}, c=0$ из (14) следует, что величина

$$
\gamma(0, \mathbf{a}, \mathbf{p})=-\ln \sum_{k=1}^{r} p_{k}\left(\frac{a_{k}}{p_{k}}\right)^{\sigma}=-\ln \min _{0 \leqslant x \leqslant 1} \sum_{k=1}^{r} p_{k}\left(\frac{a_{k}}{p_{k}}\right)^{x},
$$

где $\sigma=\sigma(0, \mathbf{a}, \mathbf{p}, \mathbf{p})$ - единственный на $(0,1)$ корень уравнения $\sum_{k=1}^{r} p_{k}\left(a_{k} / p_{k}\right)^{x} \ln \left(a_{k} / p_{k}\right)=0$, совпадает с расстоянием Чернова [27] $\rho(\mathbf{a}, \mathbf{p})=\rho(\mathbf{p}, \mathbf{a})$ между а и $\mathbf{p}$. Дифференцируя тождество (12) и учитывая знаки производных функции $\gamma(c)-c$, найдем: $V^{\prime}(d)<0, V^{\prime \prime}(d)>0$ для $d \in(0, I(\mathbf{p}, \mathbf{a}))$. Выпуклость функции $V(d)$ на $[0, I(\mathbf{p}, \mathbf{a})]$ доказана B [24].

\section{3. Построение $J$-оптимальных последовательностей крите-} риев.

Теорема 1. Последовательность байесовских критериев $\left\{\omega^{n}(\mu)\right\}$, где $\mu=\left(\mu_{1}, \ldots, \mu_{m}\right), \mu_{t}=|J|^{-1}$ для $t \in J, \mu_{t}=0$ для $t \in\{1, \ldots, m\} \backslash J$, J-оптимальна для каждого из множеств $N=A^{0}, P_{1}, P$ при $I \neq \varnothing$, $I \subseteq J$ c $M(N, J)=\infty$, если $|J|=1, u c$

$$
M(N, J)=M_{J}=\min _{i, j \in J, i<j} \rho\left(\mathbf{p}_{i}, \mathbf{p}_{j}\right), \quad \text { eслu }|J|>1 .
$$


Д ок а за т е л ь с т о. Утверждение теоремы имеется в [16] и является простым следствием утверждений из $[18$, замечание 8$]$ (или из [33, теоремы $3.1,3.2]$, [39]).

3 а м е ч а н и е 2 . Из (1), (4) при $|J|>1$ следуют оценки

$$
\alpha_{s}\left(\omega^{n}(\mu)\right) \leqslant \sum_{t \in J \backslash\{s\}} \mathbf{P}_{n}\left(\omega\left(\mathbf{p}_{t}, \mathbf{p}_{s}, 0\right), \mathbf{p}_{s}\right) \quad \text { для } s \in J .
$$

Далее можно использовать оценки из п. 2.

Теорема 2. Если $I \backslash J \neq \varnothing$, то

$$
M(P, J) \leqslant M_{I J}= \begin{cases}\delta(I, J), & \text { eсли }|J|=1 \\ \min \left(\delta(I, J), M_{J}\right), & \text { eсли }|J|>1,\end{cases}
$$

где $\delta(I, J)=\min _{t \in I \backslash J} \min _{s \in J} I\left(\mathbf{p}_{t}, \mathbf{p}_{s}\right)$.

Д ок а за т е л ь с т в о. Пусть $\left\{\omega^{n}\right\} \in P$. С учетом теоремы 1 достаточно доказать, что $M\left(\left\{\omega^{n}\right\}, J\right) \leqslant \delta(I, J)$. Для всех достаточно больших $n$ величина $1-\alpha_{t}\left(\omega^{n}\right)>0$ при всех $t \in I$. Следовательно, $\omega_{t}^{n} \neq \varnothing$ при всех $t \in I$ и $\omega_{s}^{n} \subset \Omega_{n}$ при всех $s \in J$ для всех достаточно больших $n$.

Используя оценки (см. [35, теорема 2.1], [20, § 8.4], [18, с. 298])

$$
C_{r} n^{-(r-1) / 2} \leqslant \mathbf{P}_{n}(\omega, \mathbf{p}) \exp \left(n \min _{\mathbf{u} \in \omega \cap \Omega_{n}} I(\mathbf{u}, \mathbf{p})\right) \leqslant C_{n+r-1}^{r-1},
$$

где $\omega \subseteq \Omega, \omega \cap \Omega_{n} \neq \varnothing, C_{r}$ - известная положительная константа, получим равенства

$$
\begin{aligned}
\lim _{n \rightarrow \infty}\left(n^{-1} \ln \left(1-\alpha_{t}\left(\omega^{n}\right)\right)+\min _{\mathbf{u} \in \omega_{t}^{n}} I\left(\mathbf{u}, \mathbf{p}_{t}\right)\right) & =0, \quad t \in I, \\
& \lim _{n \rightarrow \infty}\left(n^{-1} \ln \alpha_{s}\left(\omega^{n}\right)+\min _{\mathbf{u} \in \Omega_{n} \backslash \omega_{s}^{n}} I\left(\mathbf{u}, \mathbf{p}_{s}\right)\right)=0, \quad s \in J .
\end{aligned}
$$

Из (15) и из равенств $\lim n^{-1} \ln \left(1-\alpha_{t}\left(\omega^{n}\right)\right)=0$ при $t \in I$ следует, что

$$
\lim _{n \rightarrow \infty} \min _{\mathbf{u} \in \omega_{t}^{n}} I\left(\mathbf{u}, \mathbf{p}_{t}\right)=0 \quad \text { для всех } t \in I .
$$

Пусть $\min _{\mathbf{u} \in \omega_{t}^{n}} I\left(\mathbf{u}, \mathbf{p}_{t}\right)=I\left(\mathbf{u}_{t}^{n}, \mathbf{p}_{t}\right)$ при некотором $\mathbf{u}_{t}^{n} \in \omega_{t}^{n}$ для всех достаточно больших $n$. Докажем, что

$$
\lim _{n \rightarrow \infty} \mathbf{u}_{t}^{n}=\mathbf{p}_{t}
$$

Если (18) не выполняется, то существуют $\varepsilon>0$ и стремящаяся к $\infty$ последовательность $n_{1}, n_{2}, \ldots$ натуральных чисел такие, что $\left\|\mathbf{u}_{t}^{n}-\mathbf{p}_{t}\right\| \geqslant \varepsilon$ для $n=n_{1}, n_{2}, \ldots(\|\cdot\|$ - евклидова норма). Тогда в силу свойства расстояния Кульбака-Лейблера $\min _{\mathbf{u} \in \omega_{t}^{n}} I\left(\mathbf{u}, \mathbf{p}_{t}\right)=I\left(\mathbf{u}_{t}^{n}, \mathbf{p}_{t}\right) \geqslant$ 
$\min _{\mathbf{u} \in \Omega,\left\|\mathbf{u}-\mathbf{p}_{t}\right\| \geqslant \varepsilon} I\left(\mathbf{u}, \mathbf{p}_{t}\right)>0$ для $n=n_{1}, n_{2}, \ldots$, что противоречит равенству (17).

При любом $s \in J$ из (16) следует, что для всех достаточно больших $n$ величина

$$
\begin{gathered}
n^{-1} \ln \alpha_{s}\left(\omega^{n}\right)=\varepsilon_{s, n}-\min _{\mathbf{u} \in \Omega_{n} \backslash \omega_{s}^{n}} I\left(\mathbf{u}, \mathbf{p}_{s}\right)=\varepsilon_{s, n}-\min _{t \in\{0,1, \ldots, m\} \backslash s} \min _{\mathbf{u} \in \omega_{t}^{n}} I\left(\mathbf{u}, \mathbf{p}_{s}\right) \\
\geqslant \varepsilon_{s, n}-\min _{t \in I \backslash J} I\left(\mathbf{u}_{t}^{n}, \mathbf{p}_{s}\right) \\
\lim _{n \rightarrow \infty} \varepsilon_{s, n}=0 .
\end{gathered}
$$

Пусть $s(n) \in J$ и $\min _{t \in I \backslash J} I\left(\mathbf{u}_{t}^{n}, \mathbf{p}_{s(n)}\right)=\min _{s \in J} \min _{t \in I \backslash J} I\left(\mathbf{u}_{t}^{n}, \mathbf{p}_{s}\right)$. Тогда

$$
T\left(\omega^{n}, J\right) \geqslant n^{-1} \ln \alpha_{s(n)}\left(\omega^{n}\right) \geqslant \varepsilon_{s(n), n}-\min _{s \in J} \min _{t \in I \backslash J} I\left(\mathbf{u}_{t}^{n}, \mathbf{p}_{s}\right) .
$$

Отсюда и из (18), (19) следует, что

$\liminf _{n \rightarrow \infty} T\left(\omega^{n}, J\right) \geqslant-\min _{s \in J} \min _{t \in I \backslash J} I\left(\mathbf{p}_{t}, \mathbf{p}_{s}\right)=-\delta(I, J), \quad M\left(\left\{\omega^{n}\right\}, J\right) \leqslant \delta(I, J)$.

3 а м е ч а н и е 3 . Примеры показывают, что при $|J|>1$ возможны оба случая: $M_{J}<\delta(I, J), M_{J} \geqslant \delta(I, J)$. Величина, аналогичная величине $\delta(I, J)$, характеризует в [43, теорема 1] некоторую оптимальную последовательность критериев для различения двух сложных гипотез общего вида.

Лемма 3. Пусть $I \backslash J \neq \varnothing, K=I \cup J, \varepsilon_{1}, \varepsilon_{2}, \ldots-$ сходящаяся $\kappa 0$ последовательность неотрииательньх чисел. Тогда последовательность байесовских критериев $\left\{\omega^{n}\left(\lambda_{n}\right)\right\}$, где

$$
\begin{aligned}
\lambda_{n} & =\left(\lambda_{1, n}, \ldots, \lambda_{m, n}\right), \quad \lambda_{t, n}=\Lambda_{n} \quad \text { длg } t \in I \backslash J, \\
\lambda_{t, n} & =\Lambda_{n} \exp \left(n\left(M_{I J}-\varepsilon_{n}\right)\right) \quad \partial \Omega s t \in J, \\
\lambda_{t, n} & =0 \quad \partial \Omega g t \in\{1, \ldots, m\} \backslash K, \\
\Lambda_{n}^{-1} & =|I \backslash J|+|J| \exp \left(n\left(M_{I J}-\varepsilon_{n}\right)\right) \quad \text { npu } n=1,2, \ldots,
\end{aligned}
$$

имеет следуюшие свойства:

$$
\begin{aligned}
\alpha_{s}\left(\omega^{n}\left(\lambda_{n}\right)\right) \leqslant & \sum_{t \in J \backslash\{s\}} \mathbf{P}_{n}\left(\omega\left(\mathbf{p}_{t}, \mathbf{p}_{s}, 0\right), \mathbf{p}_{s}\right) \\
& +\sum_{t \in I \backslash J} \mathbf{P}_{n}\left(\omega\left(\mathbf{p}_{t}, \mathbf{p}_{s}, M_{I J}-\varepsilon_{n}\right), \mathbf{p}_{s}\right)
\end{aligned}
$$

$($ при $|J|=1$ первая сумма равна 0$)$, если $s \in J$

$$
\begin{aligned}
\alpha_{t}\left(\omega^{n}\left(\lambda_{n}\right)\right) \leqslant & \sum_{s \in J} \mathbf{P}_{n}\left(\omega\left(\mathbf{p}_{s}, \mathbf{p}_{t}, \varepsilon_{n}-M_{I J}\right), \mathbf{p}_{t}\right) \\
& +\sum_{s \in(I \backslash\{t\}) \backslash J} \mathbf{P}_{n}\left(\omega\left(\mathbf{p}_{s}, \mathbf{p}_{t}, 0\right), \mathbf{p}_{t}\right)
\end{aligned}
$$


$($ рри $|I \backslash J|=1$ вторая сумма равна 0$)$, если $t \in I \backslash J$;

$$
\limsup _{n \rightarrow \infty} T\left(\omega^{n}\left(\lambda_{n}\right), J\right) \leqslant-M_{I J}
$$

Д о к а з а т е л ь с т в о. Оценки (20), (21) следуют из (1), (4). При $s \in J$, используя оценки (5), (11) и учитывая неравенства $0<M_{I J}<$ $\theta_{2}\left(\mathbf{p}_{t}, \mathbf{p}_{s}\right)$ при любых $t \in I \backslash J, s \in J$, получим из (20) неравенства

$$
\begin{aligned}
\alpha_{s}\left(\omega^{n}\left(\lambda_{n}\right)\right) & \leqslant \sum_{t \in J \backslash\{s\}} \exp \left(-n \rho\left(\mathbf{p}_{t}, \mathbf{p}_{s}\right)\right)+\sum_{t \in I \backslash J} \exp \left(-n\left(M_{I J}-\varepsilon_{n}\right)\right) \\
& \leqslant(|J|-1) \exp \left(-n M_{J}\right)+|I \backslash J| \exp \left(-n\left(M_{I J}-\varepsilon_{n}\right)\right) \\
& \leqslant(|K|-1) \exp \left(-n\left(M_{I J}-\varepsilon_{n}\right)\right) .
\end{aligned}
$$

Отсюда следует (22).

Теорема 3. В условиях леммь 3 последовательность $\left\{\omega^{n}\left(\lambda_{n}\right)\right\}$ является $J$-оптимальной (с $\left.M(N, J)=M_{I J}\right)$ для множеств $N=P, P_{1}$ при дополнительном условии $\lim _{n \rightarrow \infty} n \varepsilon_{n}^{2}=\infty$, а при дополнительном условии $|J|>1, M_{J}<\delta(I, J), \varepsilon_{n}=0$ для $n \geqslant 1-u$ для множеств $N=P, P_{1}$,

$$
P_{0}=\left\{\left\{\omega^{n}\right\} \in A^{0}: \alpha_{t}\left(\omega^{n}\right) \leqslant(|K|-1) \exp \left(-n v_{t}\right) \partial \Omega s t \in I, n \geqslant 1\right\},
$$

гдe

$$
v_{t}=\min \left(\min _{s \in J} \gamma\left(-M_{J}, \mathbf{p}_{s}, \mathbf{p}_{t}\right), \min _{i \in(I \backslash\{t\}) \backslash J} \rho\left(\mathbf{p}_{i}, \mathbf{p}_{t}\right)\right)>0,
$$

если $t \in I \backslash J u|I \backslash J|>1$,

$$
\begin{aligned}
v_{t} & =\min _{s \in J} \gamma\left(-M_{J}, \mathbf{p}_{s}, \mathbf{p}_{t}\right)>0, \quad \text { ecлu } I \backslash J=\{t\}, \\
v_{s} & =\min \left(\min _{t \in J \backslash\{s\}} \rho\left(\mathbf{p}_{t}, \mathbf{p}_{s}\right), \min _{t \in I \backslash J} \gamma\left(M_{J}, \mathbf{p}_{t}, \mathbf{p}_{s}\right)\right) \geqslant M_{J}, \quad \text { ecлu } s \in J .
\end{aligned}
$$

Д о к а з а т е л ь с т в о. При $|I \backslash J|>1$ оценка (5) влечет неравенство

$$
\mathbf{P}_{n}\left(\omega\left(\mathbf{p}_{s}, \mathbf{p}_{t}, 0\right), \mathbf{p}_{t}\right) \leqslant \exp \left(-n \rho\left(\mathbf{p}_{s}, \mathbf{p}_{t}\right)\right)
$$

для $s, t \in I \backslash J, s \neq t$. Если $I\left(\mathbf{p}_{t}, \mathbf{p}_{s}\right)>M_{I J}$ при некоторых $t \in I \backslash J$, $s \in J$, то, применяя оценку (5), получим

$$
\begin{aligned}
\mathbf{P}_{n}\left(\omega\left(\mathbf{p}_{s}, \mathbf{p}_{t}, \varepsilon_{n}-M_{I J}\right), \mathbf{p}_{t}\right) & \leqslant \mathbf{P}_{n}\left(\omega\left(\mathbf{p}_{s}, \mathbf{p}_{t}, \varepsilon_{n}-M_{I J}\right), \mathbf{p}_{t}\right) \\
& \leqslant \exp \left(-n \gamma\left(-M_{I J}, \mathbf{p}_{s}, \mathbf{p}_{t}\right)\right) .
\end{aligned}
$$

Пусть $J_{t}=\left\{s: s \in J, I\left(\mathbf{p}_{t}, \mathbf{p}_{s}\right)=M_{I J}\right\}$ для $t \in I \backslash J$. Если $M_{J} \geqslant$ $\delta(I, J), t \in I \backslash J, s \in J_{t}$, то оценка (7) дает неравенство

$$
\mathbf{P}_{n}\left(\omega\left(\mathbf{p}_{s}, \mathbf{p}_{t}, \varepsilon_{n}-M_{I J}\right), \mathbf{p}_{t}\right) \leqslant \exp \left(-n \varepsilon_{n}^{2} h\left(\mathbf{p}_{s}, \mathbf{p}_{t}, \mathbf{p}_{t}\right)\right) .
$$


При любом $t \in I \backslash J$ с помощью (21), (24)-(26) имеем оценку

$$
\begin{aligned}
\alpha_{t}\left(\omega^{n}\left(\lambda_{n}\right)\right) \leqslant & \sum_{s \in(I \backslash\{t\}) \backslash J} \exp \left(-n \rho\left(\mathbf{p}_{s}, \mathbf{p}_{t}\right)\right) \\
& +\sum_{s \in J \backslash J_{t}} \exp \left(-n \gamma\left(-M_{I J}, \mathbf{p}_{s}, \mathbf{p}_{t}\right)\right) \\
& +\sum_{s \in J_{t}} \exp \left(-n \varepsilon_{n}^{2} h\left(\mathbf{p}_{s}, \mathbf{p}_{t}, \mathbf{p}_{t}\right)\right) .
\end{aligned}
$$

Из оценок (23), (27) следует, что $\left\{\omega^{n}\left(\lambda_{n}\right)\right\} \in P_{1}$, если $\lim _{n \rightarrow \infty} n \varepsilon_{n}^{2}=\infty$. Пусть $M_{J}<\delta(I, J)$ и все $\varepsilon_{n}=0$. Тогда $J_{t}=\varnothing$ при всех $t \in I \backslash J$; учитывая это и (5), (11), (20), (27), убеждаемся в справедливости включения $\left\{\omega^{n}\left(\lambda_{n}\right)\right\} \in P_{0}$. Отсюда, из теоремы 2 и из (22) следует, что при $N=P$, $b=M_{I J}, g^{n}=\omega^{n}\left(\lambda_{n}\right)$ верно (2). Итак, последовательность $\left\{\omega^{n}\left(\lambda_{n}\right)\right\}$ является $J$-оптимальной для $P$. Учитывая свойство $2 J$-оптимальных последовательностей, делаем вывод: $\left\{\omega^{n}\left(\lambda_{1}\right)\right\}$-оптимальна для $P_{0}, P_{1}$ при выполнении соответствующих дополнительных условий.

Теорема 4. $B$ условиях леммь 3 последовательность $\left\{\omega^{n}\left(\lambda_{n}\right)\right\}$ является $J$-оптимальной ( $\left.M(N, J)=M_{I J}\right)$ для множества $N=P$, если $M_{I J}=\delta(I, J)$ и последовательность $\left\{\varepsilon_{n}\right\}$ удовлетворяет дополнительному условию $\lim _{n \rightarrow \infty} n \varepsilon_{n}^{2}=g$, где $g$ таково, ито

$$
\max _{t \in I \backslash J, J_{t} \neq \varnothing} \sum_{s \in J_{t}} \exp \left(-g h\left(\mathbf{p}_{s}, \mathbf{p}_{t}, \mathbf{p}_{t}\right)\right)<1
$$

u $J_{t}$ определено выце при доказательстве теоремы 3.

Это утверждение доказывается аналогично предыдущему. Условие (28) гарантирует при всех достаточно больших $n$ выполнение неравенства $\alpha_{t}\left(\omega^{n}\left(\lambda_{n}\right)\right) \leqslant \alpha$ при некотором $\alpha \in(0,1)$ для всех $t \in I \backslash J$, что с учетом (23) влечет принадлежность $\left\{\omega^{n}\left(\lambda_{n}\right)\right\}$ к $P$.

3 а м е ч а н и е 4 . Утверждения теорем 3 (случай $\varepsilon_{n}=0$ для $n \geqslant 1$ ) и 4 дополняют анонсированные в [16] результаты для $P, P_{1}$.

Лемма 4. Если $I \backslash J \neq \varnothing, N \subseteq\left\{\left\{\omega^{n}\right\} \in A^{0}: \alpha_{t}\left(\omega^{n}\right) \leqslant a_{t} \exp \left(-n v_{t}\right)\right.$, $\left.a_{t}>0,0<v_{t} \leqslant \min _{s \in J} I\left(\mathbf{p}_{s}, \mathbf{p}_{t}\right) \partial \Omega s t \in I \backslash J, n \geqslant 1\right\}, m o$

$$
M(N, J) \leqslant \min _{s \in J} \min _{t \in I \backslash J} V\left(v_{t}, \mathbf{p}_{t}, \mathbf{p}_{s}\right)<\delta(I, J) .
$$

(Функиия $V$ определена в п. 2 перед леммой 1.)

Д ок а з а т е л ь с т в о. Последнее неравенство следует из убывания функции $V(x)$. Пусть $\left\{\omega^{n}\right\} \in N, s \in J, t \in I \backslash J$. Тогда

$$
\alpha_{s}\left(\omega^{n}\right)=\mathbf{P}_{n}\left(\Omega_{n} \backslash \omega_{s}^{n}, \mathbf{p}_{s}\right) \geqslant \mathbf{P}_{n}\left(\omega_{t}^{n}, \mathbf{p}_{s}\right), \quad \alpha_{s}\left(\omega^{n}\right) \geqslant \min \mathbf{P}_{n}\left(\omega_{t}^{n}, \mathbf{p}_{s}\right)
$$

при условии, что вероятность $\mathbf{P}_{n}\left(\Omega_{n} \backslash \omega_{t}^{n}, \mathbf{p}_{t}\right) \leqslant a_{t} \exp \left(-n v_{t}\right)$. 
Отсюда при любом $\varepsilon \in\left(0, v_{t}\right)$ и любом натуральном $n \geqslant \varepsilon^{-1} \ln a_{t}$

$$
\max _{s \in J} \alpha_{s}\left(\omega^{n}\right) \geqslant \min \mathbf{P}_{n}\left(\omega_{t}^{n}, \mathbf{p}_{s}\right)
$$

при условии, что вероятность $\mathbf{P}_{n}\left(\Omega_{n} \backslash \omega_{t}^{n}, \mathbf{p}_{t}\right) \leqslant \exp \left(-n\left(v_{t}-\varepsilon\right)\right)$, и

$$
\liminf _{n \rightarrow \infty} T\left(\omega^{n}, J\right) \geqslant \lim _{n \rightarrow \infty} \inf n^{-1} \ln S\left(\mathbf{p}_{t}, \mathbf{p}_{s}, v_{t}-\varepsilon\right),
$$

где $S\left(\mathbf{p}_{t}, \mathbf{p}_{s}, d\right)=\min \mathbf{P}_{n}\left(\omega, \mathbf{p}_{s}\right)$ при условии, что вероятность $\mathbf{P}_{n}\left(\omega, \mathbf{p}_{t}\right) \geqslant$ $1-\exp (-n d)$. Известно (см. [29, теорема 1 , следствие 2], [32, теорема 1]), что

$$
\lim _{n \rightarrow \infty} n^{-1} \ln S\left(\mathbf{p}_{t}, \mathbf{p}_{s}, d\right)=-V\left(d, \mathbf{p}_{t}, \mathbf{p}_{s}\right) \quad \text { для } d \in\left(0, I\left(\mathbf{p}_{s}, \mathbf{p}_{t}\right)\right) .
$$

Применяя этот результат, получим из $(29): M(N, J) \leqslant V\left(v_{t}-\varepsilon, \mathbf{p}_{t}, \mathbf{p}_{s}\right)$. Так как функция $V(d)=V\left(d, \mathbf{p}_{t}, \mathbf{p}_{s}\right)$ непрерывна (см. п. 2 ), то $M(N, J) \leqslant$ $V\left(v_{t}, \mathbf{p}_{t}, \mathbf{p}_{s}\right)$ при любых $s \in J, t \in I \backslash J$, что равносильно утверждению леммы.

Теорема 5. Пусть $I \backslash J \neq \varnothing, K=I \cup J, M_{I J}=\delta(I, J)$,

$$
\begin{gathered}
Q \in \Delta=\left(-\min _{s \in J} \min _{t \in I \backslash J} I\left(\mathbf{p}_{s}, \mathbf{p}_{t}\right), \delta(I, J)\right) ; \\
v_{t}=\min \left(\min _{s \in J} \gamma\left(-Q, \mathbf{p}_{s}, \mathbf{p}_{t}\right), \min _{i \in(I \backslash\{t\}) \backslash J} \rho\left(\mathbf{p}_{i}, \mathbf{p}_{t}\right)\right),
\end{gathered}
$$

если $t \in I \backslash J,|I \backslash J|>1, v_{t}=\min _{s \in J} \gamma\left(-Q, \mathbf{p}_{s}, \mathbf{p}_{t}\right)$, если $I \backslash J=\{t\}$,

$$
v_{s}=\min \left(\min _{t \in I \backslash J} \gamma\left(Q, \mathbf{p}_{t}, \mathbf{p}_{s}\right), \min _{j \in J \backslash\{s\}} \rho\left(\mathbf{p}_{j}, \mathbf{p}_{s}\right)\right),
$$

если $s \in J,|J|>1, v_{s}=\min _{t \in I \backslash\{s\}} \gamma\left(Q, \mathbf{p}_{t}, \mathbf{p}_{s}\right)$, если $J=\{s\}$;

$$
M=\min _{s \in J} \min _{t \in I \backslash J} \gamma\left(Q, \mathbf{p}_{t}, \mathbf{p}_{s}\right), \quad V=\min _{s \in J} \min _{t \in I \backslash J} V\left(v_{t}, \mathbf{p}_{t}, \mathbf{p}_{s}\right) .
$$

Тогда верны следующие утверждения:

1) $v_{t}>0$ длs $t \in K, v_{t}<\min _{s \in J} I\left(\mathbf{p}_{s}, \mathbf{p}_{t}\right) \partial \Omega s t \in I \backslash J, v_{s}<$ $\min _{t \in I \backslash J} I\left(\mathbf{p}_{t}, \mathbf{p}_{s}\right) \partial \Omega s s \in J, M>0, V<\delta(I, J)$;

2) последовательность байесовских критериев $\left\{\omega^{n}\left(\mu_{n}\right)\right\}$, где $\mu_{n}=$ $\left(\mu_{1, n}, \ldots, \mu_{m, n}\right), \mu_{t, n}=M_{n} \stackrel{\text { def }}{=}(|I \backslash J|+|J| \exp (n Q))^{-1} \partial \Omega s t \in I \backslash J, \mu_{t, n}=$ $M_{n} \exp (n Q)$ для $t \in J, \mu_{t, n}=0$ для $t \in\{1, \ldots, m\} \backslash K$, принадлежит множеству

$$
P_{0}=\left\{\left\{\omega^{n}\right\} \in A^{0}: \alpha_{t}\left(\omega^{n}\right) \leqslant(|K|-1) \exp \left(-n v_{t}\right) \partial \Omega s t \in I, n \geqslant 1\right\}
$$

$u$

$$
V \geqslant M\left(P_{0}, J\right) \geqslant-\liminf _{n \rightarrow \infty} T\left(\omega^{n}\left(\mu_{n}\right), J\right) \geqslant-\limsup _{n \rightarrow \infty} T\left(\omega^{n}\left(\mu_{n}\right), J\right) \geqslant M ;
$$

3) если $M=\min _{s \in J} \gamma\left(Q, \mathbf{p}_{t}, \mathbf{p}_{s}\right), v_{t}=\min _{s \in J} \gamma\left(-Q, \mathbf{p}_{s}, \mathbf{p}_{t}\right)$ при некотором $t \in I \backslash J$, mо $\left\{\omega^{n}\left(\mu_{n}\right)\right\}-J$-оптимальна для $P_{0}$ и $M\left(P_{0}, J\right)=$ $M=V$. 
Д о к аз а т ельс т в о. 1) При любых $t \in I \backslash J, s \in J$ с учетом неравенств $-I\left(\mathbf{p}_{s}, \mathbf{p}_{t}\right)<Q<I\left(\mathbf{p}_{t}, \mathbf{p}_{s}\right),(6),(9)$, (10) имеем: $0<$ $\gamma\left(Q, \mathbf{p}_{t}, \mathbf{p}_{s}\right)<I\left(\mathbf{p}_{t}, \mathbf{p}_{s}\right), 0<\gamma\left(-Q, \mathbf{p}_{s}, \mathbf{p}_{t}\right)<I\left(\mathbf{p}_{s}, \mathbf{p}_{t}\right)$. Отсюда и из неравенства $V\left(d, \mathbf{p}_{t}, \mathbf{p}_{s}\right)<I\left(\mathbf{p}_{t}, \mathbf{p}_{s}\right)$ при $d>0$ следует утверждение 1$)$.

2) Из (1), (3), (5) следуют оценки: если $t \in I \backslash J$, то (при $|I \backslash J|=1$ вторая сумма равна 0$)$

$$
\begin{aligned}
\alpha_{t}\left(\omega^{n}\left(\mu_{n}\right)\right) & \leqslant \sum_{s \in J} \exp \left(-n \gamma\left(-Q, \mathbf{p}_{s}, \mathbf{p}_{t}\right)\right)+\sum_{s \in(I \backslash\{t\}) \backslash J} \exp \left(-n \rho\left(\mathbf{p}_{s}, \mathbf{p}_{t}\right)\right) \\
& \leqslant(|K|-1) \exp \left(-n v_{t}\right) ;
\end{aligned}
$$

если $s \in J$, то (при $|J|=1$ первая сумма равна 0)

$$
\begin{aligned}
\alpha_{s}\left(\omega^{n}\left(\mu_{n}\right)\right) & \leqslant \sum_{t \in J \backslash\{s\}} \exp \left(-n \rho\left(\mathbf{p}_{t}, \mathbf{p}_{s}\right)\right)+\sum_{t \in I \backslash J} \exp \left(-n \gamma\left(Q, \mathbf{p}_{t}, \mathbf{p}_{s}\right)\right) \\
& \leqslant(|K|-1) \exp \left(-n v_{s}\right) \leqslant(|K|-1) \exp \left(-n \min _{s \in J} v_{s}\right) .
\end{aligned}
$$

При $|J|>1$ имеем: $\min _{s \in J} v_{s}=\min \left(M, M_{J}\right)$. Так как $M<\delta(I, J) \leqslant M_{J}$, то $\min _{s \in J} v_{s}=M$. Это равенство верно и при $|J|=1$. Следовательно, $\left\{\omega^{n}\left(\mu_{n}\right)\right\} \in P_{0}$,

$$
M\left(P_{0}, J\right) \geqslant-\liminf _{n \rightarrow \infty} T\left(\omega^{n}\left(\mu_{n}\right), J\right) \geqslant-\limsup _{n \rightarrow \infty} T\left(\omega^{n}\left(\mu_{n}\right), J\right) \geqslant M .
$$

Оценка $M\left(P_{0}, J\right) \leqslant V$ следует из леммы 4 .

3) Из (8), (12) имеем при некоторых $s \in J, t \in I \backslash J$ :

$$
\begin{aligned}
v_{t} & =\min _{s \in J} \gamma\left(-Q, \mathbf{p}_{s}, \mathbf{p}_{t}\right)=\min _{s \in J} \gamma\left(Q, \mathbf{p}_{t}, \mathbf{p}_{s}\right)-Q=M-Q, \\
M & =\gamma\left(Q, \mathbf{p}_{t}, \mathbf{p}_{s}\right)=V\left(\gamma\left(Q, \mathbf{p}_{t}, \mathbf{p}_{s}\right)-Q, \mathbf{p}_{t}, \mathbf{p}_{s}\right)=V\left(M-Q, \mathbf{p}_{t}, \mathbf{p}_{s}\right) \\
& =V\left(v_{t}, \mathbf{p}_{t}, \mathbf{p}_{s}\right) \geqslant V .
\end{aligned}
$$

Отсюда и из 2) следует утверждение 3 ).

3 а м е ч а н и е 5. Условия утверждения 3) выполняются при любом $Q \in \Delta$, если $|I \backslash J|=1$. Если $|I \backslash J|>1, Q<\delta(I, J)$ и близко к $\delta(I, J)$, то они выполняются для некоторого $t$ такого, что $(s, t) \in W$,

$$
W=\left\{(s, t): s \in J, t \in I \backslash J, \delta(I, J)=I\left(\mathbf{p}_{t}, \mathbf{p}_{s}\right)\right\} .
$$

Для доказательства достаточно учесть, что $\gamma\left(\delta(I, J), \mathbf{p}_{t}, \mathbf{p}_{s}\right)=\delta(I, J)$, $\gamma\left(-\delta(I, J), \mathbf{p}_{t}, \mathbf{p}_{s}\right)=0$ при $(s, t) \in W, \gamma\left(\delta(I, J), \mathbf{p}_{t}, \mathbf{p}_{s}\right)>\delta(I, J)$ при $(s, t) \notin W$ (см. п. 2). Заметим также, что если $v_{t}<\min _{s \in J} \gamma\left(-Q, \mathbf{p}_{s}, \mathbf{p}_{t}\right)$ для всех $t \in I \backslash J$ (это верно, например, при $m=3, Q=0, I=\{2,3\}$, $\left.J=\{1\}, \rho\left(\mathbf{p}_{2}, \mathbf{p}_{3}\right)<\min \left[\rho\left(\mathbf{p}_{1}, \mathbf{p}_{2}\right), \rho\left(\mathbf{p}_{1}, \mathbf{p}_{3}\right)\right]\right)$, то

$$
M \leqslant \gamma\left(Q, \mathbf{p}_{t}, \mathbf{p}_{s}\right)=V\left(\gamma\left(-Q, \mathbf{p}_{s}, \mathbf{p}_{t}\right), \mathbf{p}_{t}, \mathbf{p}_{s}\right)<V\left(v_{t}, \mathbf{p}_{t}, \mathbf{p}_{s}\right)
$$

для всех $s \in J, t \in I \backslash J, M<V$.

3 а м е ч а н и е 6 . Из свойства 2 (см. п. 1) следует, что любая $J$-оптимальная последовательность из теорем $1,3-5$ является также 
$J$-оптимальной последовательностью для некоторого множества $P_{*}$ при надлежащем выборе параметров $\alpha_{t}, n_{0}$.

Автор благодарен В.П. Чистякову, А.М. Зубкову и рецензенту за полезные замечания.

\section{СПИСОК ЛИТЕРАТУРЫ}

1. Бакут П.А. О теоретико-информационном подходе к задачам статистических решений. - Проблемы передачи информации, 1971 , т. 7 , в. 1, с. 51-57.

2. Блекуэлл Д., Гиршик М. А. Теория игр и статистических решений. М.: ИЛ, 1958, $374 \mathrm{c}$

3. Боровков A. A. Теория вероятностей. М.: Наука, 1986, 431 с.

4. Боровков A.A., Могульский $A$. $A$. Большие уклонения и проверка статистических гипотез. Новосибирск: Наука, 1992, 224 с. (Труды Ин-та математики СО PAH, т. 19.)

5. Вайда И. Оценка минимальной вероятности ошибки при проверке конечного или счетного числа гипотез. - Проблемы передачи информации, 1968, т. 4, в. 1, с. 9 19.

6. Вайда И. О сходимости средней условной энтропии. - Идентификация и аппаратура для статистических исследований: Труды Первого Всесоюзного симпозиума по статистическим проблемам в технической кибернетике. М.: Наука, 1970, c. $137-141$.

7. Вальд А. Статистические решающие функции. - Позиционные игры. Под ред. Н. Н. Воробьева и И. Н. Врублевской. М.: Наука, 1967, с. 300-522.

8. Карманов В.Г. Математическое программирование. М.: Наука, 1980, 256 с.

9. Ковалевский $B$. А. Задача распознавания образов с точки зрения математической статистики. - Читающие автоматы и распознавание образов. Киев: Наукова думка, 1965 , с. 8-37.

10. Коваль В. Н., Кук Ю. В. Проверка многих гипотез с помощью оптимальной расширенной нерандомизированной процедуры. - Украін. матем. журн., 1997, т. 49, № 8, c. $1042-1054$.

11. Кульбак $C$. Теория информации и статистика. М.: Наука, 1967, 408 с.

12. Леман Э. Проверка статистических гипотез. М.: Наука, 1964, 498 с.

13. Ляпунов A.A. О выборе из конечного числа законов распределения. - Успехи матем. наук, 1951 , т. 6 , в. 1 , с. 176-186.

14. Рао С. Р. Линейные статистические методы. М.: Наука, 1968, 548 с.

15. Розовский Л. В. Одно обобшение теоремы Колмогорова о законе повторного логарифма. - Теория вероятн. и ее примен., 1997, т. 42, в. 1, с. 134-143.

16. Салихов Н.П. Асимптотические свойства вероятностей ошибок критериев для различения нескольких мультиномиальных схем испытаний. - Докл. АН СССР, 1973 , т. 209 , № 1 , с. $54-57$.

17. Салихов Н. П. Уточнение неравенства Г. Чернова. - Теория вероятн. и ее примен., 1992 , т. 37 , в. 3, с. 583-586.

18. Салихов Н. П. Об одном обобщении расстояния Чернова. - Теория вероятн. и ее примен., 1998 , т. 43 , в. 2 , с. $294-314$.

19. Санов И. Н. О вероятности больших отклонений случайных величин. - Матем. сб., 1957, т. 2, № 1, с. 11-44.

20. Фано Р. Передача информации. Статистическая теория связи. М.: Мир, 1965, $438 \mathrm{c}$.

21. Хазен Э. М. Методы оптимальных статистических решений и задачи оптимального управления. М.: Советское радио, 1968, 256 с.

22. Хазен Э. М. Определение потенциальной точности решения некоторых задач распознавания и оценивания. - Изв. АН СССР. Техн. кибернет., 1971, № 3, с. 184192. 
23. Anderson J.A. Discrimination between $k$ population with constraints on the probabilities of misclassification. - J. Roy. Statist. Soc. Ser. B, 1969, v. 31, № 1, p. 123-139.

24. Blahut R. E. Hypothesis testing and information theory. - IEEE Trans. Inform. Theory, 1974 , v. 20 , № 4, p. 405-417.

25. Bunke O. Über optimal Verfaren der Diskriminanzanalyse. - Abh. Deutschen Akad. Wiss. Berlin. Kl. Math., Phys. und Techn., 1964, v. 4, p. 35-41.

26. Bunke O. Stabilität statistischer Entscheidungsprobleme und Anwendungen in der Diskriminanzanalyse. - Z. Wahrscheinlichkeitstheor. verw. Geb., 1967, v. 7, № 2, p. $131-146$.

27. Chernoff $H$. A measure of asymptotic efficiency for tests of a hypothesis based on the sum of observations. - Ann. Math. Statist., 1952, v. 23, № 4, p. 493-507.

28. Chernoff $H$. Large-sample theory: parametric case. - Ann. Math. Statist., 1956, v. 27 , № 1 , p. 1-22.

29. Csiszár I., Longo G. On the error exponent for source coding and for testing simple statistical hypotheses. - Studia Sci. Math. Hungar., 1971, v. 6, № 1-2, p. 181-191.

30. Efron B. The power of the likelihood ratio test. - Ann. Math. Statist., 1967, v. 38, № 3, p. 802-806.

31. Ferguson T. S. Mathematical Statistics. A Decision Theoretic Approach. New York: Academic Press, 1967, 396 p.

32. Fu F.-W., Shen S.-Y. Hypothesis testing for arbitrarily varying source with exponential-type constraint. - IEEE Trans. Inform. Theory, 1998, v. 44, № 2, p. 892895.

33. Ghosh J. K., Subramanyam K. Inference about separated families in large samples. Sankhyā Ser. A, 1975, v. 37, № 4, p. 502-513.

34. Hoeffding $W$. Probability inequalities for sums of bounded random variables. - J. Amer. Statist. Assoc., 1963, v. 58, № 301, p. 13-30.

35. Hoeffing $W$. Asymptotically optimal tests for multinomial distributions. - Ann. Math. Statist., 1965, v. 36, № 2, p. 369-408.

36. Hoel P. G., Peterson R. P. A solution to the problem of optimum classification. Ann. Math. Statist., 1949, v. 20, № 3, p. 433-438.

37. Krafft O., Plachky D. Bounds for the power of likelihood ratio tests and their asymptotic properties. - Ann. Math. Statist., 1970, v. 41, № 5, p. 1646-1654.

38. Krafft O., Puri M. L. The asymptotic behavior of the minimax risk for multiple decision problems. - Sankhyā Ser. A, 1974, v. 36, № 1, p. 1-12.

39. Leang C.C., Johnson D. H. On the asymptotics of $M$-hypothesis Bayesian detection. - IEEE Trans. Inform. Theory, 1997, v. 43, № 1, p. 280-282.

40. von Mises $R$. On the classification of observation data into distinct groups. - Ann. Math. Statist., 1945, v. 16, № 1, p. 68-73.

41. Perez $A$. Information method in reducing complex decision problems. - Transactions of the Fourth Prague Conference on Information Theory, 1965, p. 55-87.

42. Perez $A$. Information-theoretic risk estimates in statistical decision. - Kybernetika (Prague), 1967, v. 3, № 1, p. 1-21.

43. Plachky D., Steinebach J. A generalization of a result of Chernoff in large sample theory. - Math. Operationsforsch. Statist. Ser. Statist., 1977, v. 8, № 3, p. 375-379.

44. Raghavachari $M$. On theorem of Bahadur on the rate of convergence of test statistics. - Ann. Math. Statist., 1970, v. 41, № 5, p. 1695-1699.

45. Rao $C$. R. Efficient estimates and optimum inference procedures in large samples. J. Roy. Statist. Soc. Ser. B, 1962, v. 24, p. 46-63; Discussion, p. 63-72.

46. Vajda $I$. On the statistical decision problems with discrete parameter space. - Kybernetika (Prague), 1967, v. 3, № 2, p. 110-126.

47. Vajda $I$. On the amount of information contained in a sequence of independent observations. - Kybernetika (Prague), 1970, v. 6, № 4, p. 306-323.

48. Welch B. L. Note on discriminant functions. - Biometrika, 1939, v. 31, p. 218-220.

Поступила в редакцию

24.VIII. 1998

Исправленный вариант

13.III. 2000 